H. Sarbadhikari and S. M. Srivastava, Random theorems in topology

P. Loustaun au, A splitting theorem for $\mathscr{F}$-products. . 73-83

P. Pudlák, A note on bounded arithmetic . . . . . . . . . . . . . . . . . . . . . $85-89$

B. Klemp and D. Simson, On the Auslander-Reiten valued quiver of right peak

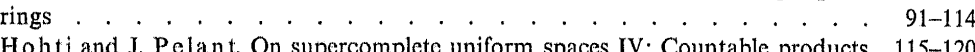

121-125

R. Pol, An n-dimensional compactum which remains $n$-dimensional after removing all

Cantor $n$-manifolds . . . . . . . . . . . . . . . 127-131

The FUNDAMENTA MATHEMATICAE publishes papers devoted to Set Theory, Topology, Mathematical Logic and Foundations, Measure and Integration,

Abstract Algebra

Each volume consists of three separate issues

Manuscripts and correspondence should be addressed to:

$$
\text { FUNDAMENTA MATHEMATICAE }
$$

Śniadeckich 8, P.O. Box 137, 00-950 Warszawa, Poland, telex PL 816112

Papers for publication should be submitted in two typewritten (double spaced) copies and contain a short abstract. A complete list of all handwritten symbols with indications for the printer should be enclosed. Special typefaces should be indicated according to the following code: script letters by encircling the typed Roman letter in black, German letters - by typing the Roman equivalent and underlining in green, boldface letters - by straight black underlining. The authors will receive 50 reprints of their articles.

Correspondence concerning subscriptions, library exchange and back numbers should be sent to:

INSTITUTE OF MATHEMATICS, POLISH ACADEMY OF SCIENCES Śniadeckich 8, P.O. Box 137, 00950 Polationa, Poland, telex PL 816112

(c) Copyright by Instytul intematy 1990

Published by PWN - Polish Scientific Publishers

ISBN 83-01-10221-7 ISSN 0016-2736

\section{Random theorems in topology}

by

\section{H. Sarbadhikari and S. M. Srivastava (Calcutta)}

Abstract. Let $E$ and $X$ be Polish spaces and $A$ and $B$ be two disjoint analytic subsets of $E \times X$ with closed vertical sections. We prove the following results.

(i) There is a Borel map $f: E \times X \rightarrow[0,1]$ such that $f \equiv 0$ on $A, f \equiv 1$ on $B$ and for each $\in E$, the map $x \rightarrow f(e, x)$ is continuous.

(ii) If $Z$ is a retract of finite ór countable product of intervals and if $f: A \rightarrow Z$ is a Borel map such that for every $e \in E$, the map $x \rightarrow f(e, x)$ is continuous then there is a Borel measurable extension $F: E \times X \rightarrow Z$ of $f$ such that $x \rightarrow F(e, x)$ is continuous for each $e \in E$.

(iii) If $A$ is Borel then (ii) holds for all convex subsets $Z$ of a second countable affine space of type $m$.

1. Notation. For notation and basic results in Descriptive Set Theory we follow Moschovakis [11]. Throughout $X$ is a Polish space with a bounded metric $d$. For $x \in X$ and positive real number $r, S_{r}(x)$ (resp. $\bar{S}_{r}(x)$ ) denotes the open (resp. closed) ball of $X$ with centre $x$ and radius $r$. Let $E$ be an arbitrary set and $\mathscr{E}$ a family of subsets of $E$. A multifunction $F: E \rightarrow X$ is a map with domain $E$ and values non-empty, closed subsets of $X$. We say that the multifunction $F: E \rightarrow X$ is $\mathscr{E}$-measurable if

$$
F^{-1}(U)=\{e \in E: F(e) \cap U \neq \varnothing\}
$$

belongs to $\mathscr{E}$ for every open set $U$ in $X$. The set

$$
\{(e, x) \in E \times X: x \in F(e)\}
$$

will be called the Graph of $F$ and will be denoted by $G(F)$. We consider a point map also as a multifunction.

Let $Z$ be a topological space and $f: G(F) \rightarrow Z$ a point map. We call $f$ a $G$-Carathéodory map if

(i) for each $e \in E, x \rightarrow f(e, x)$ is continuous and

(ii) for every $\mathscr{E}$-measurable selector $s: E \rightarrow X$ of $E$, the map $e \rightarrow f(e, s(e))$ is $\mathscr{E}$-measurable.

Let $\mathscr{E}$ be a $\sigma$-field and $G \subseteq E \times X$. Then a map $f: G \rightarrow Z$ will be called $\mathscr{E}$-Carathéodory or simply Carathéodory if for each $e \in E$, the map $x \rightarrow f(e, x)$ defined on the section $G(e)$ of $G$ is continuous and $f$ is $\mathscr{E} \times \mathscr{B}_{X} \mid G$-measurable, where $\mathscr{B}_{X}$ is the Borel $\sigma$-field of $X$ and $\mathscr{E} \times \mathscr{B}_{X}$ is the product $\sigma$-field.

1980 Mathematics Subject Classification: Primary 03E15 54C60, 54C65. 
Remark. If $Z$ is a metrizable space and $(E, \mathscr{E})$ a measurable space, then for every $\mathscr{E}$-measurable multifunction $F: E \rightarrow X$, each $G$-Carathéodory map $f: G(F) \rightarrow Z$ is Carathéodory.

Proof. By [9], fix a sequence $\left\{s_{i}\right\}$ of $\mathscr{E}$-measurable selectors of $F$ such that for every $e \in E,\left\{s_{i}(e)\right\}$ is dense in $F(e)$. Let $C$ be a closed set in $Z$ and for each positive integer $n$, let

$$
C_{n}=\left\{z \in Z: \operatorname{dist}\left(z^{\prime}, z\right)<1 / n \text { for some } z^{\prime} \in C\right\} .
$$

Then for every $(e, x) \in G(F)$,

$$
f(e, x) \in C \Leftrightarrow \forall n \exists i\left(\operatorname{dist}\left(x, s_{i}(e)\right)<1 / n \text { and } f\left(e, s_{i}(e)\right) \in C_{n}\right) .
$$

The rest of our notation is standard. If $E$ is a metrizable space then unless otherwise mentioned, $\mathscr{E}$ will denote its Borel $\sigma$-field.

For concepts in General Topology we follow Dugundji [6].

2. Introduction. Motivated by results proved in $[1,4,7]$ in [13] we proved, among others, the following two results.

THEOREM 1. Let $(E, \mathscr{E})$ be a measurable space, $F: E \rightarrow X$ an $\mathscr{E}$-measurable multifunction and $f: G(F) \rightarrow \boldsymbol{R}$ a Carathéodory map. Then there is a Carathéodory map $g: E \times X \rightarrow \boldsymbol{R}$ which extends $f$ and which satisfies

$$
g(e, X) \subseteq \operatorname{co}(f(\{e\} \times F(e))), \quad e \in E,
$$

where $\operatorname{co}(A)$ denotes the convex hull of $A$.

THEOREM 2. Let $E$ be a second countable metrizable space, $Z$ a locally convex topological vector space, $F: E \rightarrow X$ a measurable multifunction and $f: G(F) \rightarrow Z$ a G-Carathéodory map. Then also the conclusions of Theorem 1 hold.

In this paper we give generalizations of these two theorems when $E$ is a Polish space. While proving Theorem 1 we needed some random analogues of the Urysohn Theorem. Here we study this in detail and also show that our random Urysohn theorems are sharp. At the end we prove a random analogue of Lusin's theorem and raise several open problems.

\section{Random Urysohn theorems.}

THEOREM 3. If $(E, \mathscr{E})$ is a measurable space and $F_{0}, F_{1}: E \rightarrow X$ measurable multifunctions with $G\left(F_{0}\right) \cap G\left(F_{1}\right)=\varnothing$ then there is a Carathéodory map $f: E \times X \rightarrow[0,1]$ such that $f \equiv 0$ on $G\left(F_{0}\right)$ and $f \equiv 1$ on $G\left(F_{1}\right)$.

Proof. By [9], we get sequences $\left\{f_{n}^{0}\right\}$ and $\left\{f_{n}^{1}\right\}$ of measurable maps from $E$ into $X$ such that for every $e \in E,\left\{f_{n}^{\prime \prime}(e)\right\}$ is dense in $F_{c}(e)$ where $\varepsilon=0$ or 1 . Now we define

$$
\begin{aligned}
f(e, x) & =\frac{\operatorname{dist}\left(x, F_{0}(e)\right)}{\operatorname{dist}\left(x, F_{0}(e)\right)+\operatorname{dist}\left(x, F_{1}(e)\right)}, \quad(e, x) \in E \times X \\
& =\frac{\inf _{n} d\left(x, f_{n}^{0}(e)\right)}{\inf _{n} d\left(x, f_{n}^{0}(e)\right)+\inf _{n} d\left(x, f_{n}^{1}(e)\right)} .
\end{aligned}
$$

The map $f$ has the desired properties.
We give an example to show that Theorem 3 cannot be extended to the case $\mathscr{E}=\mathscr{L}_{\sigma}$ where $\mathscr{L}$ is a field.

EXAMPLE 1. Let $E=\omega^{\omega}$ and $\mathscr{E}=\Sigma_{2}^{0}$. Let $A_{0}$ and $A_{1}$ be two disjoint $\Sigma_{2}^{0}$-sets in the space of irrationals $\omega^{\omega}$ such that there do not exist disjoint $\Pi_{2}^{0}$-sets $C_{0}$ and $C_{1}$ satisfying $A_{0} \subseteq C_{0}$ and $A_{1} \subseteq C_{1}\left[11\right.$, p. 205]. Define $F_{0}, F_{1}: \omega^{\omega} \rightarrow[0,1]$ by

$$
\begin{array}{ll}
F_{0}(\alpha)=[0,3 / 4] & \text { if } \alpha \in A_{0}, \\
F_{0}(\alpha)=\{0\} & \text { if } \alpha \in \omega^{\omega} \backslash A_{0}, \\
F_{1}(\alpha)=[1 / 4,1] & \text { if } \alpha \in A_{1}, \\
F_{1}(\alpha)=\{1\} & \text { if } \alpha \in \omega^{\omega} \backslash A_{1} .
\end{array}
$$

Then $F_{0}$ and $F_{1}$ are two $\Sigma_{2}^{0}$-measurable, compact-valued multifunctions with $G\left(F_{0}\right) \cap G\left(F_{1}\right)=\varnothing$. If possible suppose there is a map $f: \omega^{\omega} \times[0,1] \rightarrow[0,1]$ such that $f \equiv 0$ on $G\left(F_{0}\right)$ and $f \equiv 1$ on $G\left(F_{1}\right)$ and such that for every $x \in[0,1], e \rightarrow f(e, x)$ is $\Sigma_{2}^{0}$-measurable. Now consider

$$
C_{0}=\left\{\alpha \in \omega^{\omega}: f(\alpha, 1 / 2)=0\right\}, \quad C_{1}=\left\{\alpha \in \omega^{\omega}: f(\alpha, 1 / 2)=1\right\} .
$$

These are disjoint $\Pi_{2}^{0}$-sets such that $A_{i} \subseteq C_{i}, i=0,1$. This is a contradiction.

For semi-continuous multifunctions we have

Lemma. Let $E$ be a metrizable space, $X$ a Polish space and $F: E \rightarrow X$ a closed valued upper or lower semi-continuous multifunction. Then for each $x \in X$ the map

$$
e \rightarrow \operatorname{dist}(x, F(e))
$$

is $\Sigma_{2}^{0}$-measurable.

Proof. Fix $x \in X, e \in E$ and reals $a<b$. We have

$$
\operatorname{dist}(x, F(e))<b
$$

$$
\begin{aligned}
& \Leftrightarrow S_{b}(x) \cap F(e) \neq \varnothing \\
& \Leftrightarrow(\exists m)\left(\bar{S}_{b-1 / m}(x) \cap F(e) \neq \varnothing\right), \\
& \operatorname{dist}(x, F(e))>a \\
& \Leftrightarrow(\exists m)\left(S_{a+1 / m}(x) \cap F(e)=\varnothing\right)
\end{aligned}
$$$$
\Leftrightarrow(\exists m)\left(\bar{S}_{a+1 / m}(x) \cap F(e)=\varnothing\right) \text {. }
$$

Equivalences (i) and (iii) prove the lemma when $F$ is lower semi-continuous. For upper semi-continuous $F$ we use (ii) and (iv).

We now have

THeOREM 4. Let $E$ be a metrizable space and $F_{0}, F_{1}: E \rightarrow X$ be lower or upper semi-continuous multifunctions such that $F_{0}(e) \cap F_{1}(e)=\varnothing$ for every $e \in E$. Then there is a map $f: E \times X \rightarrow[0,1]$ such that

(i) $f \equiv 0$ on $F_{0}$, and $f \equiv 1$ on $F_{1}$,

(ii) $x \rightarrow f(e, x)$ is continuous for every $e \in E$, and

(iii) $e \rightarrow f(e, x)$ is $\Sigma_{2}^{0}$-measurable, for each $x \in X$ 
Before we proceed to prove our next theorem we present an example.

EXAMPLE 2. Let $A$ be a $\Sigma_{1}^{1}$ but non-Borel subset of $[0,1]$. Fix a metric $\varrho$ on $\omega^{\omega}$ and let $\alpha, \beta$ be two distinct points of $\omega^{\omega}$. Let $U$ be a non-empty clopen subset of $S_{(1 / 2)(\alpha, \beta)}(\alpha)$. Let $B$ be a Borel subset of $[0,1] \times U$ with closed sections such that $A=\operatorname{proj}(B)$. Let

$$
F=B \cup([0,1] \times\{\beta\}) .
$$

Then $F$ is a Borel subset of $[0,1] \times \omega^{\omega}$ with non-empty closed sections. If possible suppose the map $e \rightarrow \operatorname{dist}(\alpha, F(e))$ defined on $[0,1]$ is Borel. Then, as

$$
e \in A \Leftrightarrow \operatorname{dist}(\alpha, F(e)) \leqslant \frac{1}{2} \varrho(\alpha, \beta),
$$

$A$ is Borel. Therefore, the map $e \hookrightarrow \operatorname{dist}(\alpha, F(e))$ is not Borel.

The above example shows that the simple-minded arguments contained in the proofs of Theorems 3 and 4 do not work for our main random Urysohn theorem mentioned in the abstract. Instead we shall use the following three results.

Theorem A (Saint-Raymond, [12]). Let $E$ and $X$ be two Polish spaces and $A$ and $B$ be two $\Sigma_{1}^{1}$-subsets of $E \times X$ such that for every $e \in E, \overline{A(e)} \cap B(e)=\varnothing$. Then there is a Borel set $C$ in $E \times X$ such that for every $e \in E, C(e)$ is closed and $A \subseteq C \subseteq(E \times X) \backslash B$.

Theorem B (Dellacherie, [5]). If $E$ and $X$ are Polish spaces and $B \subseteq E \times X$ is a Borel set with $B(e)$ open for every $e \in E$ then

$$
B=\bigcup_{n \in \omega}\left(B_{n} \times U_{n}\right)
$$

where $B_{n}$ is Borel in $E$ and $U_{n}$ open in $X$.

THEOREM C (Miller, [10]). Let $E$ be a second countable metrizable space. Denote by $\mathscr{T}$ the topology on $E$. Then given any sequence $\left\{B_{n}\right\}$ of Borel sets in $E$ there is a second countable metrizable topology $\mathscr{T}^{\prime}$ on $E$ such that

(i) each of $B_{n} \in \mathscr{T}^{\prime}$, and

(ii) the $\sigma$-fields generated by $\mathscr{T}$ and $\mathscr{T}^{\prime}$ are the same.

Actually this is a simpler case of Miller's theorem and a proof of it is also presented in $([13$, Theorem 5]).

From now on $E$ will be a Polish space.

THEOREM 5. Let $F_{0}$ and $F_{1}$ be two disjoint $\Sigma_{1}^{1}$ sets in $E \times X$ such that for each $e \in E$, the sections $F_{0}(e)$ and $F_{1}(e)$ are closed. Then there is a Carathéodory map $f: E \times X \rightarrow[0,1]$ such that $f \equiv 0$ on $F_{0}$ and $f \equiv 1$ on $F_{1}$.

Proof. By applying Theorem A twice, we get two disjoint Borel sets $C_{0}$ and $C_{1}$ in $E \times X$ with $C_{0}(e)$ and $C_{1}(e)$ closed, $F_{0} \subseteq C_{0}$ and $F_{1} \subseteq C_{1}$. By Theorem $\mathrm{B}$, we write

$$
(E \times X) \backslash C_{i}=\bigcup_{n \in \omega}\left(B_{n}^{i} \times U_{n}^{i}\right), \quad i=0 \text { or } 1
$$

with $B_{n}^{i}$ Borel in $E$ and $U_{n}^{i}$ open in $X$. Denote the topology on $E$ by $\mathscr{T}$. By Theorem C, let $\mathscr{T}^{\prime}$ be a second countable metrizable topology on $E$ such that (i) $B_{n}^{i} \in \mathscr{T}^{\prime}, n \in \omega, i=0$ or 1 , and

(ii) the $\sigma$-fields generated by $\mathscr{T}$ and $\mathscr{T}^{\prime}$ are the same.

Now $C_{0}$ and $C_{1}$ are disjoint closed sets in $E \times X$ when $E$ is equipped with $\mathscr{T}^{\prime}$ and $X$ has its own Polish topology, say $\mathscr{T}^{\prime \prime}$. By Urysohn's theorem there is a $\mathscr{T}^{\prime} \times \mathscr{T}^{\prime \prime}$-continuous map $f: E \times X \rightarrow[0,1]$ such that $f \equiv 0$ on $C_{0}$ and $f \equiv 1$ on $C_{1}$. Since the $\sigma$-fields generated by $\mathscr{T}$ and $\mathscr{T}^{\prime}$ are the same, this $f$ has all the desired properties.

Our next example shows that Theorem 5 does not hold if $F_{0}, F_{1}$ are $\Pi_{1}^{1}$.

Example 3. In Example 1 take $A_{0}$ and $A_{1}$ to be two disjoint $\Pi_{1}^{1}$ sets such that there do not exist disjoint Borel sets $C_{0}$ and $C_{1}$ with $A_{0} \subseteq C_{0}$ and $A_{1} \subseteq C_{1}$. Define $F_{0}$ and $F_{1}$ exactly the same way. The same arguments show that there does not exist a Carathéodory map $f: E \times X \rightarrow[0,1]$ such that $f \equiv 0$ on $F_{0}$ and $f \equiv 1$ on $F_{1}$.

Remark 1. It is worth noting that the following generalization of Theorem 5 also holds.

THeORem 6. Let $E$ and $X$ be Polish spaces, $F_{0}, F_{1}$ be two disjoint $\Sigma_{1}^{1}$ sets in $E \times X$ such that for all $e \in E, F_{0}(e)$ and $F_{1}(e)$ are $\Pi_{\xi}^{0}$. Then there is a Borel map $f: E \times X \rightarrow[0,1]$ such that

(i) $f \equiv 0$ on $F_{0}, f \equiv 1$ on $F_{1}$ and

(ii) for every $e \in E, x \rightarrow f(e, x)$ is $\Sigma_{\xi}^{0}$-measurable.

Proof. For $\xi=1$ this is Theorem 5. Let $1<\xi<\omega_{1}$. Embed $X$ in a recursively presented Polish space $H$, say the Hilbert cube. We now invoke a result of R. Barua ([2]) (which, in fact, is a simple extension of a result of A. Louveau [8]) and get a Borel set $B$ in $E \times X$ such that

(i) $F_{1} \subseteq B \subseteq E \times X \backslash F_{0}$, and

(ii) $B(e)$ is $\Delta_{\xi}^{0}$ for every $e \in E$.

We take $f=I_{B}$, the indicator function of $B$.

Remark 2. The argument above also works when $\xi=1$ and $X$ a zero-dimensional Polish space. In this case embed $X$ in and as a closed subspace of $\omega^{\omega}$.

4. Random extension theorems. Using the ideas contained in the proof of Theorem 5 we prove

THEOREM 7. Let $A$ be a Borel set in $E \times X$ such that the sections $A(e)$ are closed for every $e \in E$. Suppose $Z$ is a second countable convex subspace of an affine space of type $m$ and $f: A \rightarrow Z$ a Carathéodory map. Then there is a Carathéodory map g: $E \times X \rightarrow Z$ which extends $f$.

Proof. Fix a countable base $W_{1}, W_{2}, \ldots$ of $Z$. Let $A_{0}=A$ and

$$
A_{n}=A \backslash f^{-1}\left(W_{n}\right), \quad n=1,2, \ldots
$$

By the arguments contained in the proof of Theorem 5 we get a finer second countable metrizable topology $\mathscr{T}^{\prime}$ such that each of $A_{i}$ is closed when $E$ is equipped with $\mathscr{T}^{\prime}$ and the Borel $\sigma$-field of $E$ remains the same. This makes $A$ closed and $f$ continuous when $E$ has the new topology. By the extension theorem of Dugundji ([6], p. 188) there is a continuous extension $g: E \times X \rightarrow Z$ of $f$. This $g$ is a Carathéodory map when $E$ has the original topology. 
THeOREM 8. Let $A$ be a $\Sigma_{1}^{1}$ set in $E \times X$ and $Z$ a retract of a finite or countable product of intervals in $\boldsymbol{R}$. Let $f: A \rightarrow Z$ be a Borel measurable Carathéodory map. Then there is a Carathéodory map $g: E \times X \rightarrow Z$ which extends $f$.

Proof. Case 1. $Z=[-1,1]$.

We define a sequence of Carathéodory maps $g_{i}: E \times X \rightarrow[-1,1], i=0,1, \ldots$ such that for every $i$

(i) $\left|g_{i}(e, x)\right| \leqslant \frac{1}{3} \cdot\left(\frac{2}{3}\right)^{i}$, for all $(e, x)$, and

(ii) $\left|f(e, x)-g_{0}(e, x)-\ldots-g_{i}(e, x)\right| \leqslant\left(\frac{2}{3}\right)^{i}$, for all $(e, x) \in A$.

To see that such a sequence can be defined we proceed inductively. Let

$$
F_{0}^{0}=\left\{(e, x) \in A: f(e, x) \leqslant-\frac{1}{3}\right\} \quad \text { and } \quad F_{1}^{0}=\left\{(e, x) \in A: f(e, x) \geqslant \frac{1}{3}\right\} .
$$

By Theorem 5 we get a Carathéodory $g_{0}: E \times X \rightarrow[-1 / 3,1 / 3]$ having the required properties. Having defined $g_{0}, g_{1}, \ldots, g_{i}$ satisfying (i)-(iii), we let

$$
\begin{gathered}
F_{0}^{i+1}=\left\{(e, x) \in A: f(e, x)-g_{0}(e, x)-\ldots-g_{i}(e, x) \leqslant-\frac{1}{3} \cdot\left(\frac{2}{3}\right)^{i}\right\}, \\
F_{1}^{i+1}=\left\{(e, x) \in A: f(e, x)-g_{0}(e, x)-\ldots-g_{i}(e, x) \geqslant \frac{1}{3} \cdot\left(\frac{2}{3}\right)^{i}\right\} .
\end{gathered}
$$

By Theorem 5, we get a Carathéodory map $g_{i+1}: E \times X \rightarrow\left[-\frac{1}{3} \cdot\left(\frac{2}{3}\right)^{i}, \frac{1}{3} \cdot\left(\frac{2}{3}\right)^{i}\right]$ such that $g_{i+1} \equiv-\frac{1}{3} \cdot\left(\frac{2}{3}\right)^{i}$ on $F_{0}^{i+1}$ and is $\equiv \frac{1}{3} \cdot\left(\frac{2}{3}\right)^{i}$ on $F_{1}^{i+1}$.

We define

$$
g(e, x)=\lim _{i} g_{i}(e, x), \quad(e, x) \in E \times X
$$

Case 2. $Z=(-1,1)$

Using case 1, we get a Carathéodory map $h: E \times X \rightarrow[-1,1]$ which extends $f$. Let

$$
B=\{(e, x) \in E \times X:|h(e, x)|=1\} .
$$

Then $A$ and $B$ are two disjoint $\Sigma_{1}^{1}$-sets with closed sections. By Theorem 5 , we get a Carathéodory map

$$
u: E \times X \rightarrow[0,1]
$$

such that $u \equiv 1$ on $A$ and $\equiv 0$ on $B$. Put $g=u \cdot h$.

Remaining cases. It is now clear that the result is true for all intervals. When $Z$ is a finite or countable product of intervals we extend each of the coordinate functions. Finally, let $Z^{\prime}$ be a finite or countable product of intervals and $Z$ a retract of $Z^{\prime}$. Fix a retraction $r: Z^{\prime} \rightarrow Z$. If $f: A \rightarrow Z$ is a given Carathéodory map, first get a Carathéodory map $h: E \times X \rightarrow Z^{\prime}$ which extends $f$ and then take $g=r \circ h$. This completes the proof.

In Theorems 1 and 2 we get extensions satisfying

$$
g(e, X) \subseteq \operatorname{co}(f(\{e\} \times F(e))), \quad e \in E .
$$

our next example shows that we cannot have this in Theorems 7 and 8 even when $Z=\boldsymbol{R}$.
EXAMPLE 4. Let $A_{0}$ and $A_{1}$ be two $\Sigma_{1}^{1}$-sets in $[0,1]$ such that $A_{0} \cup A_{1}=[0,1]$ but there does not exist a Borel set $B$ such that $B \subseteq A_{0}$ and $B^{\mathrm{c}} \subseteq A_{1}$. Let $I_{0}$ be the space of all irrationals contained in $[0,1 / 3]$ whereas $I_{1}$ is the space of all those irrationals which are contained in $[2 / 3,1]$. Let $C_{i}$ be a Borel set in $[0,1] \times I_{i}$ whose sections are closed in $I_{i}$ and such that $\operatorname{Proj}\left(C_{i}\right)=A_{i}, i=0$ or 1 . Let $C=C_{0} \cup C_{1}$. Let $X$ be the set of all irrationals in $[0,1]$. Then we have a Borel set in $[0,1] \times X$ whose sections are closed in $X$. Define $f: C \rightarrow R$ by

$$
f(e, x)=x, \quad(e, x) \in C
$$

If possible suppose there is a Carathéodory map $g:[0,1] \times X \rightarrow \boldsymbol{R}$ which extends $f$ and which satisfies

$$
g(e, X) \subseteq \operatorname{co}(f(\{e\} \times C(e))), \quad e \in[0,1]
$$

Let

$$
B=\{e \in[0,1]: g(e, 1 / \sqrt{2}) \leqslant 1 / 2\} .
$$

Then $B$ is Borel, $B \subseteq A_{0}$ and $B^{\mathrm{c}} \subseteq A_{1}$. Contradiction.

\section{A random Lusin theorem.}

THEOREM 9. Let $f: E \times X \rightarrow[0,1]$ be a Borel map. Let $\mu(e, B)$ be a transition function on $E \times \mathscr{B}_{X}$. Then for every $\varepsilon>0$ there exists a Carathéodory map $g: E \times X \rightarrow[0,1]$ such that for every $e \in E$

$$
\mu(e,\{x \in X: g(e, x) \neq f(e, x)\})<\varepsilon .
$$

Proof. Define a sequence $\left\{E_{n}\right\}$ of subsets of $E \times X$ as follows:

$$
\begin{gathered}
E_{n}=\left\{(e, x) \in E \times X: \frac{2 k-1}{2^{n}} \leqslant f(e, x)<\frac{2 k}{2^{n}}\right. \text { for some } \\
\left.k=1,2, \ldots, 2^{n-1} \text { or } f(e, x)=1\right\} .
\end{gathered}
$$

Then $f=\sum_{n=1}^{\infty}\left(1 / 2^{n}\right) I_{E_{n}}$.

By [3], get Borel sets $F_{n}$ and $U_{n}$ in $E \times X$ such that

(i) $F_{n} \subseteq E_{n} \subseteq U_{n}, n=1,2, \ldots$;

(ii) $\mu\left(e, F_{n}(e) \backslash U_{n}(e)\right)<\varepsilon / 2^{n}$ for $n=1,2, \ldots$ and $e \in E$; and

(iii) $F_{n}$ (e) and $X \backslash U_{n}(e)$ are compact for each $n$ and $e$.

Now, $e \rightarrow F_{n}(e)$ and $e \rightarrow X \backslash U_{n}(e)$ are measurable, closed-valued multifunctions for each $n$. Hence by Theorem 3 , there exist Carathéodory maps $g_{n}: E \times X \rightarrow[0,1]$ such that

$$
g_{n}(e, x)= \begin{cases}0 & \text { if }(e, x) \in X \backslash U_{n}, \\ 1 & \text { if }(e, x) \in F_{n} .\end{cases}
$$

Put $g=\sum_{n=1}^{\infty}\left(1 / 2^{n}\right) g_{n}(e, x)$. 


\section{Open problems.}

Problem 1. In Theorem 5 suppose we take $F_{0}, F_{1}$ to be Borel but $E$ an arbitrary second countable metrizable or even a $\boldsymbol{\Pi}_{1}^{1}$-set. Do the conclusions of Theorem 5 hold in this case?

Problem 2. Does Theorem 7 hold for a $\Sigma_{1}^{1}$-set $A$ ? We do not know the answer even when $Z$ is a convex subset of $\boldsymbol{R}^{2}$.

Problem 3. Can Theorem 8 be extended for $\boldsymbol{\Pi}_{1}^{1}$-sets $A$ ? We do not know the answer even when $Z=R$

A question related to Problem 3 is the following:

Problem 4. Let $C_{0}$ and $C_{1}$ be two disjoint $\Pi_{1}^{1}$-sets in $E \times X$ such that for every $e \in E$, the sections $C_{0}(e)$ and $C_{1}(e)$ are closed. Further assume that there is a Borel set $B$ containing $C_{0}$ but disjoint from $C_{1}$. Do there exist disjoint Borel sets $B_{0}$ and $B_{1}$ such that $C_{0} \subseteq B_{0}, C_{1} \subseteq B_{1}$ and for every $e \in E$, the sections $B_{0}(e)$ and $B_{1}(e)$ are closed in $X$ ?

\section{References}

[1] G. F. Andrus and L. Brown, Measurable extension theorems, J. Math. Anal. Appl. 96 (1983), 454-462.

[2] R. Barua, Structure of hyperarithmetical sets of ambiguous Borel classes, preprint.

[3] D. Blackwell and C. Ryll-Nardzewski, Non-existence of everywhere proper conditional distributions, Ann. Statist. 34 (1963), 223-225.

[4] F. S. de Blasi and J. Myjak, On the random Dugundji extension theorem, J. Math. Anal. Appl. 128 (1987), 305-311.

[5] C. Dellacherie, Ensembles Analytiques: Théorèmes de séparation et applications, Lecture Notes in Math. 465, Springer-Verlag, Berlin-Heildelberg.

[6] J. Dugundji, Topology, Prentice Hall of India, New Delhi 1975.

[7] $\mathrm{O}$. H a ňs, Measurability of extensions of continuous random transformations, Ann. Statist. 30 (1959), 1152-1157.

[8] A. Louveau, A separation theorem for $\Sigma_{1}^{1}$ sets, Trans. Amer. Math. Soc. 260 (1980), 363-378.

[9] A. M a itra and B. V. R a o, Generalizations of Castaing's theorem on selectors, Colloq. Math. 42 (1979), 295-300.

[10] D. E. Miller, Borel selectors for separated quotients, Pacific J. Math. 91 (1980), 187-198.

[11] Y. N. Moschovakis, Descriptive Set Theory, North-Holland Publishing Company, Amsterdam-New York-Oxford.

[12] J. Saint-Raymond, Boréliens à coupe $K_{\sigma}$, Bull. Soc. Math. France 104 (1976), 389-400,

[13] H. Sarbadhikari and S. M. Srivastava, Random Tietze and Dugundji extension theorems, J. Math. Anal. Appl., submitted.

\section{STAT-MATH DIVISION}

INDIAN STATISTICAL INSTITUTE

203 B. T. Road

Calcutta 700035

Received 7 November 1988 .

in revised form 7 June 1989

\section{A splitting theorem for $\mathscr{F}$-products}

by

Philippe Loustaunau (Fairfax, Va.)

Abstract. Let $\mathscr{F}$ be a non-principal ultrafilter on an infinite index set $A$. Let $\left\{M_{a}: a \in A\right\}$ be a family of left $R$-modules. We define the $\mathscr{F}$-product of the $M_{a}$ 's to be $\prod_{a \in A}^{\mathscr{F}} M_{a}=\left\{\left(m_{a}\right)_{a \in A} \in \prod_{a \in A} M_{a}\right.$ : $\left.\left\{a \in A: m_{a}=0\right\} \in \mathscr{F}\right\}$. In the present paper, we determine a necessary condition for the $\mathscr{F}$-product of the $M$ 's to split in the corresponding direct product. This condition will be given in terms of the lattice of ideals which are annihilators of subsets of a certain factor ring of $R$, and will depend on $\mathscr{F}$.

$R$ will always denote a ring with identity, all modules will be unital, $A$ will always denote an infinite index set and $|X|$ will always denote the cardinality of a set $X$.

1. Introduction. The question of when the canonical embedding of a direct sum of modules splits in the corresponding direct product has been extensively studied. See, for example, [2], [9], [11], [18], and [19]. Recently modules that are in between direct sums and direct products, called $x$-products, have been introduced. See, for example, [5], [6], [13], [14] and [17]. In [14], a necessary condition was determined for the canonical embedding of the $x$-product of modules in the corresponding direct product to split. This result generalized the above-mentioned classical theorems on the sum-product splitting property.

The study of $x$-products can be done in a more natural setting. Indeed, the $x$-products are special cases of a larger class of submodules of the direct product, called $\mathscr{F}$-products, where $\mathscr{F}$ is a filter on the index set $A$. The main objective of this paper is to determine a necessary condition for the $\mathscr{F}$-product of modules to split in the corresponding direct product. This condition will be given in terms of the lattice of ideals which are annihilators of subsets of a certain factor ring of $R$, and will depend on $\mathscr{F}$.

Let $\operatorname{cpl}(\mathscr{F})$ be the largest cardinal number $x$ such that $\mathscr{F}$ is $x$-complete. If $\operatorname{cpl}(\mathscr{F})=\aleph_{0}$ (e.g., if $|A|<$ first measurable cardinal number) and if the $\mathscr{F}$-product splits in the corresponding direct product, then a certain factor ring of $R$ has the Ascending Chain Condition (ACC) on annihilators (Theorem 3.5). Under these hypotheses, if $R$ is simple or if $M_{a}=R$ for every $a \in A$, then $R$ itself has ACC on annihilators (Corollaries 3.6 and 3.7).

The proof of Theorem 3.5 extends a technique that was used in [2] and [14] Following the classical definition (see, for example, [1], [3], [4], [8], [12] and [16]), 